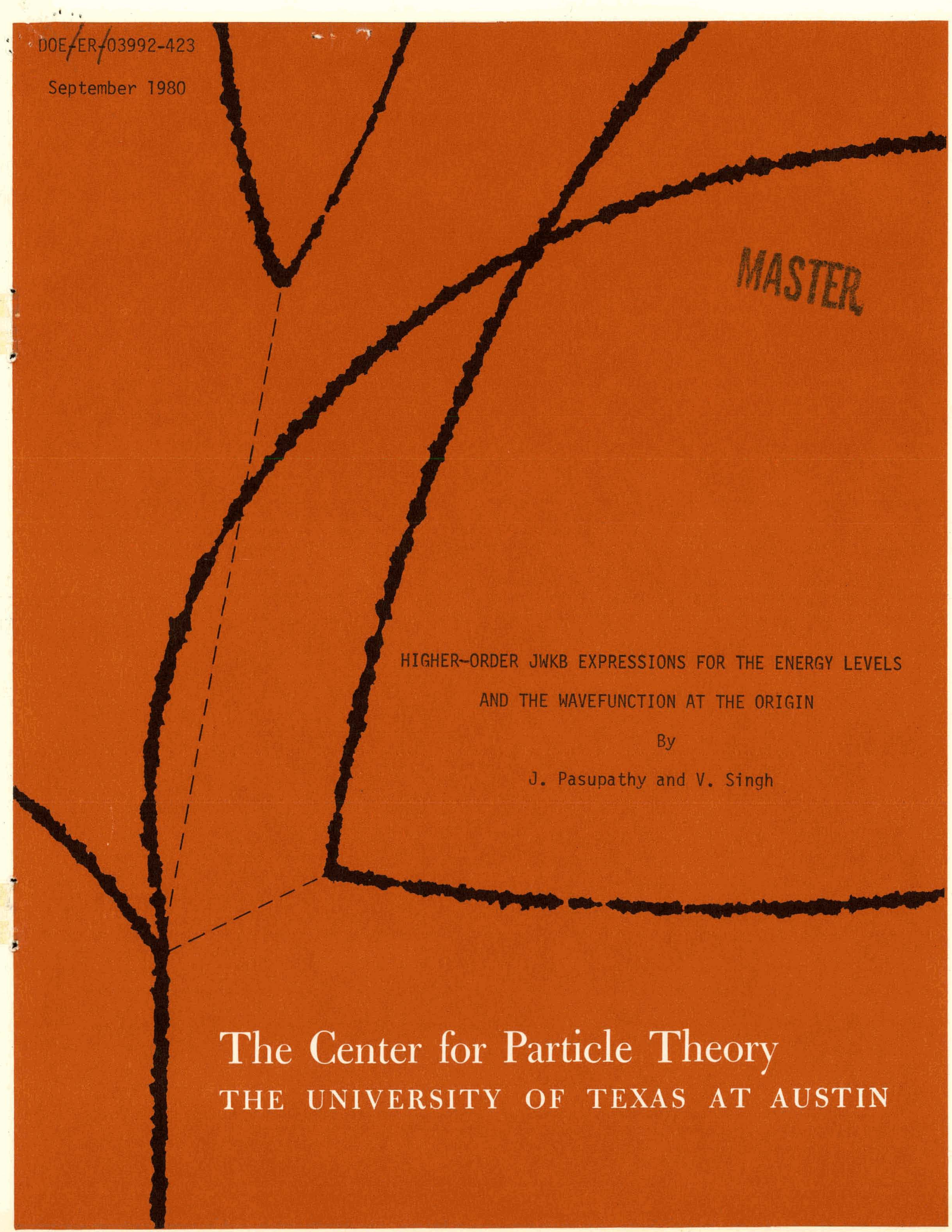




\section{DISCLAIMER}

This report was prepared as an account of work sponsored by an agency of the United States Government. Neither the United States Government nor any agency Thereof, nor any of their employees, makes any warranty, express or implied, or assumes any legal liability or responsibility for the accuracy, completeness, or usefulness of any information, apparatus, product, or process disclosed, or represents that its use would not infringe privately owned rights. Reference herein to any specific commercial product, process, or service by trade name, trademark, manufacturer, or otherwise does not necessarily constitute or imply its endorsement, recommendation, or favoring by the United States Government or any agency thereof. The views and opinions of authors expressed herein do not necessarily state or reflect those of the United States Government or any agency thereof. 


\section{DISCLAIMER}

Portions of this document may be illegible in electronic image products. Images are produced from the best available original document. 


\title{
HIGHER-ORDER JWKB EXPRESSIONS FOR THE ENERGY LEVELS \\ AND THE WAVEFUNCTION AT THE ORIGIN
}

\author{
J. Pasupathy ${ }^{\star}$ and Virendra Singh ${ }^{\dagger}$ \\ Center for Particle Theory \\ Department of Physics
}

The University of Texas at Austin

Austin, Texas 78712 U.S.A.

*Permanent Address: Centre for Theoretical Studies, Indian Institute of Science, Bangaldre- 560012 India.

TPermanent Address: Tata Institute of Fundamental Research, Colaba Bombay- 400005 India.

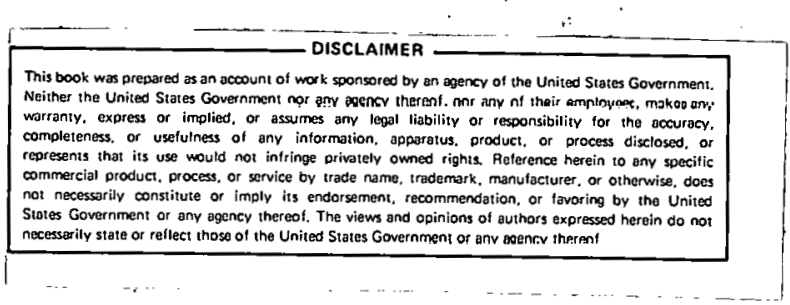




\section{Abstract}

We derive an exact quantization condition for the energy levels of a particle in a radial potential assumed finite at the origin. This is used to derive corrections to the semiclassical JWKB quantization condition. We further relate the normalization integral of the wavefunction to the energy derivative of wavefunction at origin and use this expression to derive the corrections to the semiclassical JWKB expressions for the wavefunction at origin. An application to upsilon leptonic decay width is also given. 


\section{INTRODUCTION}

Methods of non-relativistic quantum mechanics have been extensively used recently to study many of the properties of heavy quarkonium systems. 1 Although the quark anti-quark potential is, presumably, of the confining type, the potential is not known in detail. Thus for example, while the Cornell group ${ }^{2}$ has obtained a satisfactory explanation of many of the properties of the chormonium system with a Coulomb plus linear potential, Martin ${ }^{3}$ on the other hand has obtained a successful fit to the chormonium spectra with a potential of the type

$$
V(r)=A+B r \varepsilon
$$

with $\varepsilon \approx 0.1$.

Although given any $V(r)$, one can at least numerically compute to arbitrary accuracy, any quantity of interest, it is worthwhile to ask how much one can learn about the system from a knowledge of the energy levels alone as these are directly accessible from the experiment. Typical quantities of interest are $\left|r^{-\ell_{R}} R_{n \ell}(r)\right|_{r=0}^{2}$ where $R_{n \ell}(r)$ is the $n$th radial wavefunction of the Schrödinger equation for the $\ell^{\text {th }}$ partial wave. For example the value of the $S$-wave function at the origin $\left|R_{n 0}(0)\right|$ is needed to find the decay rates of orthocharmonium into lepton pairs or into 3 gluons. Similarly the derivative of the p-wave function is needed to compute the decay ratio of $\mathrm{O}^{++}, \mathrm{1}^{++}$and $2^{++}$states, then gluon-production rates in hadron collisions, as well as in diffraction production of the $1^{++}$in neutrino reactions. 
The lowest order JWKB approximation already provides a good estimate of these quantities. In this approximation one has for the S-wave function at the origin 4

$$
\left|R_{n 0}\right|^{2}=\frac{1}{\pi}\left(\frac{2 m E_{n 0}}{\hbar^{2}}\right) \frac{\partial\left(2 m E_{n 0}\right) / \hbar^{2}}{\partial n}
$$

which, generalized to all partial waves reads as 5

$$
\left|r^{-\ell} R_{n \ell}(r)\right|_{r=0}^{2}=\frac{1}{\pi}\left|\frac{\ell !}{(2 \ell+1) ! !}\right|^{2}\left(\frac{2 m E_{n \ell}}{\hbar^{2}}\right)^{\ell+\frac{1}{2}} \frac{\partial\left(2 m E_{n \ell}\right) / \hbar^{2}}{\partial n}
$$

As is well known Eq. (1.2) and (1.3) become quite accurate in the short wavelength or large $n$ limit. 6

One of the aims of this paper is to provide a more accurate version of Eq. (1). We derive an exact relation between $\left|R_{n 0}(0)\right|^{2}$ and $\frac{\partial E}{\partial n}$ which when expanded in a JWKB series yields Eq. (1.2) as the leading term. The successive terms of the series can be easily evaluated when the potential is analytic at $r=0$.

Apart from its relevance to the questions discussed above the JWKB method is of intrinsic interest by itself. For this reason, we have also found it worthwhile to write down the higher order correction to the energy levels and evaluate them explicitiy, as an illustration, for potentials of the type $V=A r^{k}$ with $k$ an integer.

When the potential is non-analytic at the origin like e.g. $V=A r^{k}$ with $k>0$ but non-integral, the various integrals that occur in the calculation need suitable regularization. To make the procedure rigorous, 
we develop an alternate expansion based on integral equation for the exact eigenfunction. This is discussed in Section IV. In Section $V$ we comment on leptonic decay widths of the upsilon levels.

\section{I1. HIGHER JWKB APPROXIMATIONS}

Introducing $u(r)=r \psi(r)$ we can write the S-wave Schrödinger equation as

$$
\frac{d^{2} u}{d r^{2}}+[\lambda-V(r)] u(r)=0 \quad 0 \leq r<\infty
$$

with $\lambda=2 m E / h^{2}, V=2 m / \hbar^{2} U(r)$ where $U(r)$ is the potential energy. Defining $V(R)=\lambda$ the solution to Eq. (2.1) in the inside region $r<R$ can be written as

$$
u(r)=\left[\exp \pm \frac{i}{\hbar} \int_{R} a(r, \lambda) d r\right] \sqrt{a(r, \lambda)}
$$

where $a(r, \lambda)$ satisfies the equation

$$
a^{2}-\frac{3 \hbar^{2}}{4 a^{2}}\left(\frac{d a}{d r}\right)^{2}+\frac{\hbar^{2}}{2 a} \frac{d^{2} a}{d r^{2}}=2 m[E-U(r)]
$$

The general solution for $r<R$ is then

$$
u(r)=\frac{c_{1}}{\sqrt{a(r, \lambda)}} \exp \left\{\frac{i}{\hbar} \int_{R}^{r} a(r, \lambda) d r\right\}+\frac{c_{2}}{\sqrt{a(r, \lambda)}} \exp \left\{-\frac{i}{\hbar} \cdot \int_{R}^{r} a(r, \lambda) d r\right\}
$$

while the outside solution for $r>R$, vanishing at infinity is 


$$
u(r)=\frac{C}{2 \sqrt{b}} \exp \left(-\frac{1}{\hbar} \int_{R}^{r} h(r, \lambda) d r\right)
$$

where b satisfies the equation

$$
b^{2}-\frac{\hbar^{2}}{2 b} b^{\prime \prime}+\frac{3 \hbar^{2}}{4}\left(\frac{d b}{d r}\right)^{2}=2 m[E-U(r)]
$$

The inside and outside solutions Eq. (2.4) and Eq. (2.5) can be matched 7 by continuing them in the complex $r$-plane for large enough $|R-r|$, with

$$
c_{1}=\frac{1}{2} C e^{i \pi / 4} \quad C_{2}=\frac{1}{2} C e^{-i \pi / 4}
$$

Hence the solution near the orjgin $(R>r \geq 0)$ is

$$
u(r, \lambda)=\frac{C}{\sqrt{a(r, \lambda)}} \sin \left(\frac{1}{\hbar} \int_{r}^{R} a(r, \lambda) d r+\frac{\pi}{4}\right)
$$

The eigenvalue condition $u(0, \lambda)=0$ reads

$$
\frac{i}{\hbar} \int_{0}^{R} a\left(r, \lambda_{n}\right) d r+\frac{\pi}{4}=n \pi
$$

We may emphasize here that the expressions (2.8)-(2.9) are exact.

One of our interests is to calculate the value of the normalized wave function at the origin

$$
|\psi(0)|^{2}=\frac{\left|u^{\prime}(0, \lambda)\right|^{2}}{\int_{0}^{\infty}\left(u(r, \lambda)^{2} d r\right.}
$$

where prime denotes differentiation with respect to $r$. Consider now 
solutions of the Schrödinger equation corresponding to arbitrary energy values $\lambda$ and $\lambda^{\prime}$ which satisfy the boundary condition at infinity but not at the origin

$$
\begin{aligned}
& u^{\prime \prime}(r, \lambda)+(\lambda-v) u(r, \lambda)=0 \\
& u^{\prime \prime}\left(r, \lambda^{\prime}\right)+\left(\lambda^{\prime}-v\right) u\left(r, \lambda^{\prime}\right)=0
\end{aligned}
$$

From Eq. (2.11) and Eq. (2.12) we deduce

$$
\frac{d}{d r}\left\{u(r, \lambda) u^{\prime}\left(r, \lambda^{\prime}\right)-u^{\prime}(r, \lambda) u\left(r, \lambda^{\prime}\right)\right\}=\left(\lambda-\lambda^{\prime}\right) u\left(r, \lambda^{\prime}\right) u(r, \lambda)
$$

Integrating Eq. (2.13) from $0 \leq r<\infty$ and letting $\lambda^{\prime}-\lambda=\lambda_{m}$ an eigenvalue we find $^{8}$

$$
\left|\psi_{m}(0)\right|^{2}=\frac{1}{4 \pi} \frac{\left[u^{\prime}\left(0, \lambda_{m}\right)\right]^{2}}{\int_{0}^{\infty}\left[u\left(r, \lambda_{m}\right)\right]^{2} d r}=\frac{1}{4 \pi} \frac{u^{\prime}\left(0, \lambda_{m}\right)}{\left.\frac{\partial u(0, \lambda)}{\partial \lambda}\right|_{\lambda=m_{m}}}
$$

This again is an exact equation. ${ }^{9}$ It takes a particularly simple and form when we substitute the formal solution Eq. (2.8) in Eq. (2.14) and use the eigenvalue condition Eq. (2.9).

$$
\left|\psi_{m}(0)\right|^{2}=\frac{1}{4 \pi^{2}} \frac{2 m}{(\hbar)^{3}} a(0, \lambda) \cdot \frac{\partial E}{\partial n}
$$

If $\frac{\partial E}{\partial n}$ is determined from the energy spectrum, the only unknown quantity is $a(0, \lambda)$ which can be written as JWKB series as follows. Returning to Eq. (2.3), we solve for $a(r, \lambda)$ as a power series in $\hbar^{2}$. writing 


$$
a(r, \lambda)=a_{0}(r, \lambda)-\hbar^{2} a_{2}(r, \lambda)+\cdots
$$

and substituting in Eq. (2.3) we find

$$
\begin{gathered}
a_{0}(r, \lambda)=p(r, \lambda)=+\sqrt{2 m[E-V(r)]} \\
a_{2}(r, \lambda)=-\frac{3}{8 a_{0}^{3}(r, \lambda)}\left(-\frac{d a_{0}(r, \lambda)}{d r}\right)^{2}+\frac{1}{4 a_{0}^{2}(r, \lambda)} \frac{d a_{0}^{2}(r, \lambda)}{d r^{2}}
\end{gathered}
$$

Substituting Eq. (2.17) we get

$$
a_{2}(r, \lambda)=-\frac{U^{\prime \prime}}{8 \sqrt{2 m}[E-U(r)]^{3 / 2}}-\frac{5}{32} \frac{\left(U^{\prime}\right)^{2}}{\sqrt{2 m}[E-U(r)]^{5 / 2}}
$$

It follows then to order $\hbar^{2}$

$$
|\psi(0)|^{2}=\frac{1}{4 \pi^{2}} \frac{(2 m)}{\hbar^{3}} \frac{\partial E}{\partial n} \sqrt{2 m E}\left[1+\frac{\hbar^{2} U^{\prime \prime}(0)}{16 m E^{2}}+\frac{5 \hbar^{2}}{32} \cdot \frac{\left(U^{\prime}(0)\right)^{2}}{2 m E^{3}}\right]
$$

It is illuminating to rewrite Eq. (2.20) as

$$
\begin{aligned}
|\psi(0)|^{2}=\frac{11}{4 \pi} & \frac{2 m}{\hbar^{3}} \frac{\partial E}{\partial n} \sqrt{2 m E} \cdot \frac{1}{2}\left[\left(1-\frac{U \cdot\left(\frac{\hbar}{\sqrt{2 m E}}\right)}{E}\right)^{-\frac{1}{4}}\right. \\
& \left.+\left(1-\frac{U\left(-\frac{\hbar}{\sqrt{2 m E}}\right)}{E}\right)^{-\frac{1}{4}}\right]
\end{aligned}
$$

This implies that the corrections to $|\psi(0)|^{2}$ are determined by the value of the potential at one de Broglie wavelength on either side of the origin. ${ }^{10}$ This is in conformity with the physical interpretation ${ }^{11}$ that Eq. (1.2) corresponds to free particle behavior over short time intervals. 
III. ENERGY EIGENVALUES

We now return to the quantization condition

$$
\frac{1}{\hbar} \int_{0}^{R} \mathrm{a}\left(r, \lambda_{n}\right) \mathrm{dr}=\left(n-\frac{1}{4}\right) \pi
$$

and substitute the JWKB series Eq. (2.16)-(2.19). We get

$$
\begin{aligned}
\left(n-\frac{1}{4}\right) \pi \hbar= & \left.\int_{0}^{R} \sqrt{2 m[E-U(r)}\right] d r+\hbar^{2} \int_{0}^{R} d r\left\{\frac{U^{\prime \prime}}{8 \sqrt{2 m}[E-U(r)]^{3 / 2}}\right. \\
& \left.+\frac{5}{32} \frac{\left(U^{\prime}\right)^{2}}{\sqrt{2 m}[E-U(r)]^{5 / 2}}\right\}+O\left[(\hbar)^{2}\right]
\end{aligned}
$$

As an illustration example consider $U=A r^{k}$ ( $k=$ positive integer). It is evident that the integrand in the correction term is ill-defined at the classical turning point $r=R$. An elementary way to regularize the integrals is to use the identity

$$
\frac{\partial^{t}}{\partial E^{t}}[E-U(r)]^{\alpha}=\alpha(\alpha-1) \cdots(\alpha-t-1)[E-U(r)]^{\alpha-t}
$$

in Eq. (2.10). For large enough integer $t$, perform the integrals over $r$ and then differentiate $t$ times. We will show, in Section IV, that the correct regularized expression is given by

$$
\left(n-\frac{1}{4}\right) \pi h=\int_{0}^{R} \sqrt{2 m(E-U(r))} d r+\frac{\hbar^{2}}{\sqrt{2 m}} \delta
$$

where 


$$
\begin{gathered}
\delta=-\frac{5}{72 \int_{0}^{R} \sqrt{E-U(r)} d r}+\frac{1}{4} \int_{0}^{R} f\left(r^{\prime}\right) \sqrt{E-U\left(r^{\prime}\right)} d r^{\prime}\left[h\left(r^{\prime}, E\right)\right]^{2}+\cdots \\
f(r)=\frac{U^{\prime \prime}(r)}{4(E-U(r))^{2}}+\frac{5}{32} \frac{\left[U^{\prime}(r)\right]^{2}}{[E-U(r)]^{3}}-\frac{5}{72} \frac{1}{\left[\int_{0}^{R}(E-U)^{\frac{1}{2}} d r\right]^{2}}
\end{gathered}
$$

and

$$
\begin{gathered}
h(r, E)=\sqrt{\frac{2 \pi z}{3}}\left\{J_{1 / 3}(z)+J_{-1 / 3}(z)\right\} \\
z(r, E)=\frac{1}{\hbar} \int_{r}^{R} \sqrt{2 m(E-U(r))} d r
\end{gathered}
$$

For potentials $U(r)$ analytic at $r=0$ we can replace $\left[h\left(r^{\prime}, E\right)\right]^{2}$ inside the integrand in (3.4) by its average value 2 . Doing that we obtain for $U=a r^{k}(k=1,2, \cdots)$ and

$$
\begin{aligned}
& E=\lambda a\left(\hbar^{2} / 2 m \dot{a}\right)^{k / k+2} \\
& \lambda^{\frac{1}{2}+\frac{1}{k}}=\frac{2\left(n-\frac{1}{2}\right) \sqrt{\pi} \Gamma\left(\frac{3}{2}+\frac{1}{k}\right)}{\Gamma\left(1+\frac{1}{k}\right)}\left\{1+\frac{(k-2)}{96 \pi} \frac{\Gamma\left(1+\frac{1}{k}\right) \Gamma\left(2-\frac{1}{k}\right)}{\Gamma\left(\frac{3}{2}+\frac{1}{k}\right) \Gamma\left(\frac{3}{2}-\frac{1}{k}\right)} \frac{1}{\left(n-\frac{1}{4}\right)^{2}}+\cdots\right\} \\
& \text { for } k \geq 2
\end{aligned}
$$

and

$$
\lambda=\left[\frac{3 \pi}{2}\left(n-\frac{1}{4}\right)\right]^{2 / 3}\left\{1+\frac{5}{48} \frac{1}{\left[\frac{3 \pi}{2}\left(n-\frac{1}{4}\right)\right]^{2}}+\cdots\right\} \quad \text { for } k=1
$$




\section{INTEGRAL EQUATION METHOD}

It is evident that, the singular behavior of the integrals in Eq. (3.1) is related to the non-analyticity of $a_{0}(r, \lambda)$ at $r=R$. Further if we consider potentials of the type $U(r)=A r^{k}$ (k = non-integral), $a_{0}(r, \lambda)$ is non-analytic at the origin as well, so that both Eq. (2.20) and Eq. (3.1) become meaningless. Since this difficulty is artificial due to the failure of the expansion Eq. (2.16) at $r=0$ and $r=R$ we now consider the integral equation form of the Schrödinger equation which is free from these troubles.

Adopting the procedure due to Langer ${ }^{12}$ and Titchmarsh ${ }^{13}$ setting $2 \mathrm{~m}=\mathrm{h}=1$ and defining

$$
\begin{aligned}
& \zeta(r)=\int_{R}^{r}[E-V(r)]^{\frac{1}{2}} d r \\
& n(r)=[E-U(r)]^{\frac{1}{4}} u(r)
\end{aligned}
$$

the Schrödinger equation can be written as

$$
\frac{d^{2} \eta}{d \zeta^{2}}+\left[1+\frac{5}{36 \zeta^{2}}\right] \eta=\hbar^{2} f(r) \eta
$$

where

$$
f(r)=\frac{5}{36} \frac{1}{\left[\int_{R}^{r}(E-U(r))^{\frac{1}{2}} d r\right]^{2}}-\frac{1}{4} \frac{U^{\prime \prime}}{(E-U)^{2}}-\frac{5}{16} \frac{\left(U^{\prime}\right)^{2}}{(E-U)^{3}}
$$


To solve (4.3) let

$$
\begin{gathered}
h(z)=\sqrt{\frac{2 \pi z(r)}{3}}\left\{J_{1 / 3}(z)+J_{-1 / 3}(z)\right\} \\
j(z)=\sqrt{\frac{\pi z(r)}{2}} J_{1 / 3}(z) .
\end{gathered}
$$

and

$$
z(r, E)=\frac{1}{f_{i}} \int_{r}^{R} \sqrt{2 m(E-V(r))} d r
$$

Then the solution which satisfies the boundary condition at infinity is

$$
n(r, E)=h(z(r, E))+\int_{r}^{\infty} G\left(r, r^{\prime} ; E\right) f\left(r^{\prime}\right) \eta\left(r^{\prime}, E\right) \sqrt{E-V\left(r^{\prime}\right)} d r^{\prime}
$$

Note that $h(z(r, E)) \rightarrow 0$ exponentially in $r$ as $r \rightarrow \infty$. The Green's function $G\left(r, r^{\prime}\right)$ is given by

$$
G\left(r, r^{\prime} ; E\right)=h\left(z\left(r^{\prime}, E\right)\right) j(z(r, E))-\left(r \leftrightarrow r^{\prime}\right) .
$$

The first order solution of the integral equation (4.5) is given by

$$
\eta(r, E)=h(z(r, E))+\int_{r}^{\infty} G\left(r, r^{\prime} ; E\right) f\left(r^{\prime}\right) h\left(z\left(r^{\prime}, E\right)\right) \sqrt{E-U\left(r^{\prime}\right)} d r^{\prime}
$$

and should be used in calculation of the energy eigenvalues as well as the calculation of the wavefunction behavior near $r=0$. The eigenvalue condition is obtained by imposing the boundary condition at $r=0$, namely the vanishing of the wavefunction there, i.e.

$$
n(0, E)=0 .
$$


This leads to the regularization mentioned earlier in the previous section. Note that the integrand in the expression (4.7) has only integrable singularities at $r=0$ or $r=\dot{R}$ and is thus perfectly well behaved. The major contribution to the integrals is, of course, from the classically accessible region $R \geq r \geq 0$ as the integrand vanishes exponentially outside that region.

\section{LEPTONIC WIDTHS OF T STATES.}

As a simple but all the same quite useful application of Eq. (1.2), consider the leptonic widths of the $15,25,35$ and $4 S$ states of the $T$ resonances. According to recent experiemnts ${ }^{14}$, the median energy values of these states is given by

$$
\begin{array}{cc}
M(T)=9.4345 \mathrm{GeV} & \Delta M(2 S-1 S)=559 \mathrm{MeV} \\
\Delta M(3 S-1 S)=889 \mathrm{MeV} & \Delta M(4 S-1 S)=1114 \mathrm{MeV}
\end{array}
$$

we shall need only the relative leptonic widths of these states which are known more accurately than their absolute values. We have

$$
\begin{gathered}
\tilde{\Gamma}_{2}=\frac{\Gamma_{2}}{\Gamma_{1}}=0.39 \pm 0.06 \quad \tilde{\Gamma}_{3}=\frac{\Gamma_{3}}{\Gamma_{1}}=0.32 \pm 0.04 \\
\tilde{\Gamma}_{4}=\frac{\Gamma_{4}}{\Gamma_{1}}=0.25 \pm 0.07 .
\end{gathered}
$$


Now the leptonic width of a vector meson is given by the Van-Royen Weisskopf formula

$$
\Gamma_{n}=\left|\psi_{n}(0)\right|^{2} \cdot \frac{16 \pi \alpha^{2} e_{Q}^{2}}{M_{n}^{2}} \cdot k
$$

where $k$ is a gluonic radiation correction factor presumed to be independent of n. Using Eq. (1.2) we can therefore write if the potential is finite at the origin

$$
\tilde{\Gamma}_{n}=B \frac{k_{n}}{M_{n}^{2}} \frac{d E}{d n}
$$

where $B$ is a constant whose value we shall not need here, with

$$
M_{n}=2 m_{q}+E_{n} \quad k_{n}^{2}=m_{q}\left(E_{n}-v(0)\right)
$$

where $V(0)$ is the value of the potential at the origin and $m_{q}$ is the quark mass ( $\mathrm{m}_{\mathrm{q}} / 2$ is the reduced mass). We calculate the derivation $\frac{\mathrm{dE}}{\mathrm{dn}}$ at $n=2,3,4$ from

$$
\begin{gathered}
\frac{d E_{2}}{d n}=\frac{1}{2}\left(E_{3}-E_{1}\right) \quad \frac{d E_{3}}{d n}=\frac{1}{2}\left(E_{4}-E_{2}\right) \\
\frac{d E_{4}}{d n}=E_{4}-E_{3}-\frac{1}{2}\left(E_{4}-E_{2}+2 E_{3}\right)
\end{gathered}
$$

According to Eq. (5.4) through (5.6)

$$
\left(\frac{\tilde{\Gamma}_{n} M_{n}^{2}}{\frac{d E}{d n}}\right)^{2}=B^{2}\left(E_{n}-\text { constant }\right)
$$


using for $\tilde{\Gamma}_{2}$ and $\tilde{\Gamma}_{3}$ their median values given in (5.2) we predict $\tilde{\Gamma}_{4}$ to be 0.23 , in satisfactory agreement with experiment. Note that the only assumption made about the potential is that it is smooth and finite at the origin.

When data on higher energy levels as well as precise measurements of the leptonic widths become available, it will become necessary to take into account the higher JWKB corrections, especially for small $n$ values. Besides it would be necessary to take into account the Coulombic corrections as discussed in ref. (15).

Acknowledgments

The authors are grateful to Prof. E. C. G. Sudarshan for valuable discussions, and to him and members of the Center for Particle Theory for their hospitality. One of us (J.P.) is grateful to Prof. J. S. Beli for valuable correspondence. 


\section{FOOTNOTES AND REFERENCES}

1. Many excellent reviews are available in the literature. See e.g. K. Gottfried in Proceedings of the 1977 International Symposium on Lepton and Photon Interactions at High Energies. Hamburg, F. Gutbrod, ed. (DESY, Hamburg (1978).) C. Quigg and J. L. Rosner, Phys. Reports $\underline{56}$, 167 (1979); H. Grosse and A. Martin, Phys. Reports 60, 343 (1980).

2. E. Eichten, K. Gottfried, T. Kinoshita, K. D. Lane and T. M. Yan, Phys. Rev. D 21, 203 (1980).

3. A. Martin, Phys. Lett. 93B, 338 (1980).

4. M. Krammer and P. Leal-Ferreira, Rev. Bras. Fis. $\underline{6}, 7$ (1976):

C. Quigg and J. L. Rosner, Phys. Rev. D 17, 2364 (1978).

5. J. S. Bell and J. Pasupathy, Phys. Lett. 83B, 389 (1979).

6. For smooth potentials the formulae seem to be good even for small $n$ values. See ref. 5. For an illustrative application of Eq. (1.2) to the $T$ system see Section $V$ of the present paper.

7. Cf. L. D. Landau and E. M. Lifshitz, "Quantum Mechanics Non-Relativistic Theory." Translated by J. B. Sykes and J. S. Bell. Second edition 1965. Pergamon Press. Chapter VII $\$ 47$.

8. The extra factor $\frac{1}{4 \pi}$ in Eq. (2.14) is the normalization factor for the angular part of the wavefunction.

9. Eq. (2.14) was derived independently by J. S. Be17. Private communication to one of us (J.P.) in 1979.

10. We have assumed $U(0)=0$; otherwise replace $E$ by $E-U(0)$ and $U\left( \pm \frac{\hbar}{\sqrt{2 \mathrm{mE}}}\right)$ $U\left( \pm \frac{\hbar}{\sqrt{2 m(E-U(0))}}\right)-U(0)$ in Eq. (2.21). Notice that a JWKB estimate 
of the correction can be obtained from the knowledge of $\frac{\mathrm{dE}}{\mathrm{dn}}$ by the inversion formula $r(V)=\frac{2 \hbar}{(2 m)^{\frac{1}{2}}} \int_{0}^{V} \frac{d E}{(V-E)^{\frac{1}{2}}}\left(\frac{d E}{d n}\right)^{-1}$.

11. See ref. 5 .

12. R. E. Langer, Phys. Rev. 51, 669 (1937).

13. E. C. Titchmarsh, "Eigenfunction Expansions," Part I, p. 151. Oxford: Clarendon Press, 1962. 2nd edition.

14. D. Andrews et a7. Phys. Rev. Lett. 45,219 (1980); G. Finocchiaro et al. ibid. p. 222.

15. J. S. Bell and J. Pasupathy, Zeit. für Phys. 2C, 183 (1979). 